Romana Radulescu

Universidad Autónoma de Madrid

\title{
CONSTRUCCIONES IDIOMÁTICAS CON EL VERBO SALIR EN ESPAÑOL, INGLÉS Y RUMANO ${ }^{1}$
}

\section{Introducción}

El objetivo de este trabajo es estudiar el comportamiento semántico del verbo salir en distintas combinaciones de palabras, principalmente las combinaciones que presentan cierto grado de fijación como lo son las construcciones idiomáticas o metafóricas. Es decir, el estudio se centrará en analizar el verbo salir desde el punto de vista de sus posibles extensiones de significado sugeridas por un importante número de expresiones idiomáticas formadas con dicho verbo, tanto en español como en otras lenguas, en nuestro caso, el inglés y el rumano. El corpus de construcciones idiomáticas y metafóricas se presentará en el apartado 2 pero adelanto aquí algunos ejemplos en español (1), en inglés (2) y en rumano (3):

(1) salir adelante, salir a pedir de boca, salir redondo, salir del agujero

(2) come out ahead, come out of a jam, turn out perfectly, come out well

(3) a iesi la liman, a o scoate la capat, a iesi pe dos, a iesi din incurcatura

De acuerdo con mi hipótesis, es posible establecer una definición básica del verbo salir en español que lo hace susceptible de adquirir muy diversos significados dependientes de los distintos contextos en los que pueda aparecer y este fenómeno no es un hecho idiosincrásico, ni determinado culturalmente, tal y como se ha considerado tradicionalmente. Para apoyar la hipótesis he considerado combinaciones de palabras con los verbos correspondientes al verbo salir en otros dos idiomas, el inglés y el rumano; el análisis intenta dar cuenta de las similitudes y las diferencias observadas.

Incluyo en el corpus de expresiones analizadas tanto expresiones idiomáticas propiamente dichas -aquellas combinaciones de palabras fijadas en la lengua, cuyo significado global no se puede deducir como suma de los significados de sus constituyentes- como expresiones metafóricas, menos estables en la lengua, pero en las que el verbo salir presenta asimismo un cambio de significado. La razón que me ha permitido unificar para este estudio las dos clases de expresiones es que tanto la formación como la interpretación de las expresiones idiomáticas son procesos ligados a mecanismos cognitivos como la metáfora o la metonimia, tal y como lo demuestran datos recientes en la bibliografía sobre expresiones idiomáticas (Ruiz Gurillo, 2001; Glucksberg, 2001; Dobrovol'skij, 2000).

Por otro lado, la investigación aborda el estudio de las expresiones metafóricas o idiomáticas desde la perspectiva del Lexicón Generativo de James Pustejovsky, de acuerdo con el cual la recuperación del significado de las combinaciones de palabras se puede atribuir a la información contenida en el léxico y a los mecanismos que regulan la buena for-

\footnotetext{
1 Este trabajo ha sido sufragado por una beca de FPI de la Comunidad e Madrid y ha sido asimismo parcialmente financiado por el proyecto «Las expresiones idiomáticas con verbos de movimiento: propuesta de elaboración de un diccionario teórico, de uso y contrastivo» (Ref. FG 05, Vicerrectorado de Investigación, UAM; investigador principal, Elena de Miguel).
} 
mación e interpretación de dichas combinaciones de palabras (J. Pustejovsky 1995, 1998, 2000). El presente estudio profundiza en esta línea de investigación y pretende demostrar que, en efecto, las expresiones idiomáticas como combinaciones estables de palabras, se forman y se interpretan de la misma manera que las combinaciones libres de palabras (Radulescu, 2004, 2005; De Miguel, 2004b, 2005) y predice, además, que los procesos y mecanismos implicado en la descodificación de las expresiones idiomáticas son independientes del idioma en que se dan.

Me sitúo, pues, en el marco teórico del Lexicón Generativo y parto de la hipótesis de que el léxico tiene una organización regular y productiva, capaz de generar extensiones de significados a partir de primitivos semánticos - en este sentido la teoría lleva el nombre de Lexicón Generativo - y de dar cuenta, de esta manera, de fenómenos como la polisemia, la metáfora o la metonimia. Además, la teoría postula la existencia de mecanismos generativos responsables de desencadenar distintos significados de las palabras dependiendo de las combinaciones en las que entren.

En otras palabras, el estudio parte del supuesto de que las palabras no tienen una definición cerrada sino que están definidas en el léxico con una definición básica y unos aspectos esenciales que las capacitan para adquirir otros sentidos, en función de la información que aportan los otros elementos léxicos con los que se combinan. Los mecanismos generativos son, pues, principios que controlan la buena formación de las combinaciones de palabras, y son responsables de los nuevos significados emergentes.

La definición de un verbo, en este caso salir, tiene que ser una definición poco específica en el sentido de que está potencialmente capacitada para desarrollar distintos significados según el contexto.

Concretamente, el análisis está basado en los conceptos de Infraespecificación, Estructura Eventiva y Estructura de Qualia ${ }^{2}$ que más adelante se presentarán de manera esquemática en los apartados 1.1-1.3.

\subsection{Infraespecificación}

El concepto básico del planteamiento de Pustejovsky es la noción de infraespecificación (en inglés, underspecification) que no es más que el grado de imprecisión (vagueness) que, según el autor, caracteriza las definiciones de las unidades léxicas y que, en su opinión, permite dar cuenta de las distintas interpretaciones que las palabras adquieren en distintos contextos.

Así pues, según Pustejovsky, se puede minimizar el número de entradas diferentes en un lexicón para dar cuenta de significados distintos pero relacionados de una unidad léxica - lo que él denomina polisemia lógica - el autor propone que en el léxico existen entradas infraespecificadas que recogen los posibles sentidos que una palabra puede adquirir en el contexto. Por ejemplo, desde esta perspectiva, no es necesario enumerar dos definiciones distintas para la palabra bueno que den cuenta de los dos significados diferentes que se pueden recuperar en los ejemplos (4) y (5):

(4) un buen paraguas

(5) un buen niño

${ }^{2}$ Pustejovsky propone otras dos estructuras, la Estructura Argumental y la Estructura de Herencia Léxica, que en conjunto forman los cuatro niveles de representación integrados en las entradas de las palabras en el léxico; razones de espacio me impiden presentarlas aquí y remito al lector a los trabajos de este autor citados en la bibliografía. 
Lo que hace posible las dos interpretaciones distintas del adjetivo bueno en los ejemplos (4) y (5) -un paraguas que funciona bien, un niño que tiene buen carácter- es la información potencial contenida en cuatros aspectos esenciales del significado. Así pues, el autor argumenta que «debemos simplemente mantener el significado de bueno suficientemente vago como para cubrir todos los casos posibles: buen tiempo, buen profesor, buena película etc.» (J. Pustejovsky, 1995:43). Pustejovsky denomina estos cuatro aspectos esenciales qualia, papeles o roles y la información estructurada en estos cuatro rasgos esenciales compone la Estructura de Qualia de las palabras que, en su opinión, es la responsable del significado de sus posibles combinaciones.

\subsection{La Estructura de Qualia}

Los cuatro tipos de quale que, según Pustejovsky, reúnen las características esenciales de una pieza léxica son: el constitutivo, el formal, el télico y el agentivo.

Así pues, la información relativa al material, al peso, a las partes y los elementos componentes de un objeto se recoge en el quale CONSTITUTIVO, que en palabras del autor, nos da la relación entre un objeto y sus partes constituyentes («gives the relation between an object and its constituents, or proper parts», Pustejovsky, 1995: 85).

La información sobre la orientación, magnitud, forma y dimensionalidad, color y posición de un objeto, se almacena en el quale FORMAL. A través de este quale, pues, se puede distinguir un objeto en un conjunto más amplio («that wich distinguishes the object within a larger domain», id.).

El quale TÉLICO informa sobre el propósito, el fin o la función del objeto («built-in function or aim», $i d$.), es decir, el propósito que el agente tiene al ejecutar o realizar alguna acción o la función intrínseca de ciertas actividades.

Por último, el quale AGENTIVO recoge la información sobre los factores implicados en el origen de un objeto («factors envolved in the origin or bringing about of an object», $i d$.), es decir, información sobre su posible creador, tipo natural o la cadena causal a través de la cual ha llegado a existir.

El caso de la palabra novela, a la que el autor le atribuye la Estructura de Qualia recogida en (7), es un ejemplo de cómo funciona en el caso de los nombres la Estructura de Qualia.

(7) novela: quale constitutivo: narración

quale formal: libro (x)

quale télico: leer (e1, $\mathrm{y}, \mathrm{x})$

quale agentivo: escribir (e2, $\mathrm{z}, \mathrm{x})$

La adecuada selección de los qualia -télico o agentivo- determinará las interpretaciones alternativas (8a) y (8b) de la frase recogida en (8):

(8) María empezó la novela.

(8a) María empezó a leer la novela. (selección del quale télico)

(8b) María empezó a escribir la novela. (selección del quale agentivo) ${ }^{3}$

\footnotetext{
3 J. Pustejovsky, 1995 apud De Miguel y Fernández Lagunilla, 2004.
} 
Así pues, el modelo de lexicón generativo de Pustejovsky intenta describir cómo se generan los diversos significados de las palabras y propone que, en lugar de enumerarlos, el lexicón debería proporcionar entradas infraespecificadas susceptibles de tomar uno de entre los múltiples y variados sentidos interrelacionados de la palabra, siempre dependiendo del contexto. Este modelo, como ya se verá más adelante, permite explicar fenómenos que, como apunta De Miguel (2004b), «se dan en todas las lenguas» y que «las explicaciones tradicionales han relegado a menudo al terreno del saber enciclopédico y del conocimiento del mundo».

\subsection{La Estructura Eventiva}

En cuanto a los eventos, Pustejovsky propone que los eventos no son entidades atómicas sino que se pueden descomponer en distintas fases o subeventos entre los cuales existe una jerarquía de prominencia, por tanto, están dotados de una estructura interna que él denomina Estructura Eventiva, en la cual se indica el tipo de evento expresado por un elemento léxico o un sintagma.

Las clases de eventos que el autor propone son estados, procesos y transiciones ${ }^{4}$. Los estados (E) son eventos simples, con duración y sin fases; por ejemplo, amar, saber, tener. Los procesos $(\mathrm{P})$ son una secuencia de subeventos idénticos, con duración; por ejemplo, correr, buscar, nadar. Por último, las transiciones (T) son eventos complejos que constan de un proceso a través del cual se alcanza un estado; por ejemplo, leer un libro, construir una casa, llegar.

La Teoría del Lexicón Generativo establece además un conjunto de principios, llamados Mecanismos Generativos, que controlan las condiciones de buena formación de los sintagmas y las oraciones y captan la relación semántica entre sus constituyentes. En otras palabras, estos mecanismos son operaciones que pueden variar el tipo inicialmente asociado a una entrada léxica según el contexto en que ésta entre ${ }^{6}$.

\subsection{Clasificación aspectual de De Miguel y Fernández Lagunilla (2000, 2004)}

En línea con la clasificación aspectual de los eventos de Vendler y dentro del marco teórico de Pustejovsky, está la propuesta de clasificación elaborada por De Miguel y Fernández Lagunilla que presupone la existencia de ocho clases distintas de eventos atendiendo a su estructura interna (De Miguel y Fernández Lagunilla, 2000a,b, 2004; De Miguel, 2004a). Por la relevancia que esta clasificación tiene para mi trabajo, haré en este apartado una breve presentación.

\footnotetext{
${ }^{4}$ Existen varias clasificaciones de verbos por su aspecto, pero la más extendida entre los lingüistas es la clasificación de Vendler (Z. Vendler, 1967, apud E. De Miguel, 1999), según sea el verbo dinámico o estático, con límite o sin límite, con duración o sin duración. Así pues, según Vendler, existen cuatro clases aspectuales de verbos: estados (eventos no dinámicos, con duración y sin límite); actividades (eventos dinámicos, con duración y sin límite); realizaciones (eventos dinámicos, con duración y límite) y logros (eventos dinámicos, con límite y sin duración).

${ }^{5}$ Por tanto, las transiciones de la clasificación de Pustejovsky incluyen las realizaciones (leer un libro) y los logros (llegar) de la clasificación de Vendler. La realización implica un proceso y un estado, mientras que un logro prescinde del subevento proceso desdencadenante del estado.

${ }^{6}$ La dimensión de este trabajo no me permite detallar los Mecanismos Generativos (el Ligamiento Selectivo, la Coerción o Coacción de Tipos y la Co-composición). Se encuentra información sobre ellos en los trabajos de Pustejovsky citados.
} 
Las autoras amplían la clasificación de Pustejovsky teniendo en cuenta la culminación de algunos eventos y las fases que preceden o siguen al punto culminante. Distinguen pues entre los logros que son eventos simples de los que son eventos complejos con una fase posterior de estado o proceso; entre los procesos distinguen un subgrupo que puede llegar a culminar y también diferencian dos clases de transiciones, entre una fase de proceso y una de logro o entre dos logros.

Desde esta perspectiva, las autoras proponen ocho clases aspectuales de verbos:

a. los estados E son eventos simples, con duración y sin fases, como por ejemplo, tener, odiar.

b. los procesos P1 son una secuencia de eventos simples idénticos, con duración y sin fases, como por ejemplo, estudiar, nadar.

c. las transiciones $\mathrm{T} 1$ son procesos o actividades que desembocan en un punto seguido de un cambio de estado, eventos delimitados, con duración que culminan en la fase final como por ejemplo, leer un libro, ver una película.

d. los logros simples L1 son eventos delimitados que ocurren en un punto, por ejemplo, explotar, llegar, nacer.

e. los logros compuestos L2 son eventos delimitados que culminan en un punto que es la fase inicial y que va seguido de un estado, por ejemplo, marearse, ocultarse, sentarse.

f. los logros compuestos L3 son eventos delimitados que culminan en un punto que es la fase inicial y que va seguido de un proceso, por ejemplo, hervir, florecer, ver la costa.

g. las transiciones T2 son eventos delimitados que implican una transición entre dos puntos de culminación; tanto el subevento inicial como el subevento final pueden a su vez descomponerse en dos fases, por ejemplo, bajar(se), caer(se), ir(se), su$\operatorname{bir}(s e)$.

h. por último, los procesos P2 son eventos incrementativos o de acabamiento gradual, por ejemplo, adelgazar, engordar, envejecer.

De manera más esquemática, se puede dar a las ocho clases de verbos la representación recogida en (9):
(9) a. E (e)
b. $\mathrm{P}(\mathrm{e} 1 \ldots \mathrm{en})$
c. T1 (P, L (L, E))
d. L1 (-E, E)
e. $\mathrm{L} 2(\mathrm{~L}, \mathrm{E})$
f. L3 (L, P)
g. T2 (L3 (L, (P)), L2 (L, (E)))
h. P2 (P, (L))

\section{Expresiones idiomáticas con el verbo salir}

Abordo ahora el tema central de este trabajo, que he tratado asimismo en R. Radulescu (2004), y comienzo por presentar los datos. 
Tanto en español como en otras lenguas, las expresiones idiomáticas o metafóricas formadas con el verbo salir alcanzan un número importante. Su composición léxica y su realización sintáctica son asimismo muy diversas. Sin embargo, para mi análisis he encontrado cuatro grandes grupos en los que se pueden dividir, según su significado global.

El Grupo A abarca aquellas expresiones, recogidas en (10), cuya interpretación idiomática se puede parafrasear grosso modo como «librarse de alguna dificultad o situación embarazosa».

El Grupo B está formado por las expresiones recogidas en (11), cuya interpretación idiomática se podría parafrasear como «apartarse». Esta interpretación se puede entender tanto en sentido físico como de actitud.

El Grupo C comprende las expresiones cuyo significado global es «aparecer, hacerse público, surgir», recogidas en (12).

El Grupo D lo forman aquellas expresiones que tienen un significado idiomático equivalente más o menos a «resultar», recogidas en (13).

(10) salir a flote

salir del paso

salir del agujero

salir del apuro

salir adelante

no saber por dónde salir

no salir de una para entrar en otra

salir del sartén y caer en las brasas

salir del cascarón

salir de su concha

(12) salir al paso

salirle de las narices

ya salió aquello

salir con esas

salir a (la) luz

salir al aire

salir [a la arena/al ruedo]

salir en la tele

salir el sol

salir en defensa

salirle los colores

salir los dientes
(11) salir(se) por la tangente

salir(se) por peteneras

salirse del tiesto

salirse de sus casillas

salirse de madre

salirse de quicio

salir [pitando/disparado/zumbando/flechado]

salir de estampida

salir por pies

(13) salir a pedir de boca

salir [redondo/bordado/bien/caro/mal]

salir [ganando/perdiendo]

salir rana

salir el tiro por la culata

salir (clavado) a alguien

salirse con la suya

salir la criada respondona

salir escaldado

a lo que salga

salir con la cabeza caliente y los pies fríos

salir tarifando 


\section{Análisis}

\section{1. ¿Qué significa el verbo salir?}

Una primera aproximación a las construcciones metafóricas o idiomáticas recopiladas para este estudio nos puede llevar a concluir que el verbo salir tiene muchas y muy diversas acepciones y así lo confirma la entrada de diccionario de salir que abarca más de una treintena de significados distintos, relacionados o no?.

El propósito de este trabajo ha sido proponer una definición básica del verbo salir que permita la recuperación del significado que éste pueda tener en distintas combinaciones de palabras, libres o estables, metafóricas o idiomáticas.

Como he anticipado, este análisis parte de la hipótesis de que los verbos cuentan con un significado básico, infraespecificado, que los hace susceptibles de adquirir nuevos significados en contexto. Asimismo, de acuerdo con De Miguel y Fernández Lagunilla (2004) y De Miguel 2004b, asumo que la especificación de los verbos en el léxico es un continuo: cuanto más específica la definición, menor la potencialidad significativa y más restricciones sintácticas. A la inversa, cuanto menor sea la especificación de la definición, mayor la potencialidad designativa y mayor el número de contextos alternativos.

Por consiguiente, parece legítimo asumir que si una palabra tiene múltiples acepciones y aparece en muy diversas combinaciones, es consecuencia de su poca carga semántica: sus significados vienen dados por las unidades léxicas con las que comparte contexto ${ }^{8}$. Éste parece ser el caso del verbo salir: su significado básico, suficientemente infraespecificado, puede ser «pasar de un lugar delimitado a uno no delimitado»?.

En los siguientes apartados analizaré en qué manera esta definición del verbo salir permite recuperar los significados globales recogidos en los grupos A, B, C y D, pero antes, demos un paso más hacia la descodificación del verbo. Si aceptamos que salir significa «pasar de un lugar a otro», independientemente de la naturaleza de los dos lugares, delimitados o no, el evento denominado por el verbo es, pues, una transición entre dos puntos culminantes.

Cada uno de estos dos puntos culminantes representa un evento simple: abandonar y llegar. El primer punto de culminación puede ir seguido o no de un proceso -el desplazamiento de un lugar a otro-, por ejemplo, en las expresiones salir pitando o salir de estampida, mientras que el segundo punto de culminación puede ir seguido o no de un estado -en el caso de la forma salirse-, como en la expresión salirse de sus casillas.

Así pues, la definición básica que hemos adoptado para salir es congruente con la configuración aspectual de una transición T2, en la clasificación de De Miguel y Fernández Lagunilla (2000b, 2004), que reproduzco en (14).

$$
\text { salir: } \quad \text { T2 (L3 (L, (P)), L2 (L, (E))) }
$$

\footnotetext{
${ }^{7}$ Según el DRAE 1999. La recuperación de todos estos sentidos a través de un posible significado básico y su adecuada co-composición con otros complementos es objeto de otro trabajo, véase Radulescu, en preparación.

${ }^{8}$ Sigo en este sentido a I. Bosque (2001). El autor defiende la existencia de una clase de verbos que en determinadas construcciones se comportan como los verbos light -de soporte o de apoyo-. La hipótesis de Bosque es la de que cuando estos verbos -que él denomina heavier light verbs- se vacían de significado, la información que conservan es meramente de tipo aspectual.

${ }^{9}$ G. Wotjak (2000) usa el término «circunscrito» en su definición del verbo salir, como ejemplo de «descripción pormenorizada» de los significados verbales: «con salir se designa un movimiento donde el arg x/AGENTE abandona un lugar/espacio circunscrito LOC/LOC Source» (G. Wotjak 2000).
} 
En los siguientes apartados detallaré asimismo qué fases del evento se enfocan en cada una de sus realizaciones semánticas, es decir, cómo se relacionan los subeventos enfocados -el logro, el proceso, el estado- con los significados globales de las expresiones idiomáticas A, B, C y D.

\subsection{A. Salir como «librarse»}

Las expresiones recogidas en (10) tienen como denominador común la interpretación idiomática de «librarse» de alguna situación difícil o incómoda. En línea con Gurillo (2001) y Glucksberg (2001), la recuperación del significado idiomático de las expresiones se hace a través de procesos metafóricos o metonímicos que, en la mayoría de los casos, han contribuido a su formación.

Asimismo, de acuerdo con G. Lakoff (1980), un lugar delimitado o un contenedor puede ser la conceptualización metafórica de una situación difícil o incómoda y por tanto, palabras como, cascarón, agujero, concha, sartén etc., representan el recipiente que, a su vez, codifica la expresión metafórica de la situación difícil. El evento denotado por salir, es decir, pasar de este contenedor a un lugar no delimitado - que puede aparecer explícitamente indicado por palabras como, a flote, adelante - se interpreta, pues, como «librarse».

La fase de la transición más probable de ser enfocada en el discurso es la fase de logro L, el subevento puntual en el que culmina la liberación: T2 (L3 (L, P), L2 $(L, \mathrm{E}))^{10}$.

\subsection{B. Salir como «apartarse»}

Las expresiones idiomáticas del Grupo B, recogidas en (11), tienen como significado global «apartarse», desde el punto de vista espacial o desde el punto de vista del comportamiento. En el primer caso, el significado de salir se aproxima más a su significado literal, de diccionario, aunque el énfasis cae más bien sobre la manera de «pasar», es decir, de desplazarse, pitando, zumbando, flechado, disparado. Estas palabras son asimismo expresiones metafóricas de la prisa: salir como de un disparo, como una flecha, como un tren etc.

La fase de la transición que los complementos de salir enfocan es, por tanto, la fase de proceso P: T2 (L3 $(\mathrm{L}, P), \mathrm{L} 2(\mathrm{~L}, \mathrm{E}))$.

En el segundo caso, nos encontramos de nuevo con la metáfora conceptual del CONTENEDOR: esta vez son las normas y las restricciones que impone un comportamiento educado o prudente, las que se interpretan como un contenedor. Las palabras casillas, tiesto, madre, quicio ponen los límites, acotan el lugar delimitado. De ahí que, insubordinarse, insolentarse o exagerar, es decir, apartarse de las reglas, normas o restricciones sea interpretado metafóricamente como salirse [del tiesto/de quicio/de madre ${ }^{11}$.

\footnotetext{
${ }^{10}$ En esta fórmula y las siguientes, la fase enfocada del evento aparece en cursiva.

${ }^{11}$ En la interpretación de las expresiones metafóricas intervienen a menudo más de una metáfora conceptual entre las cuales existen relaciones bien establecidas y coherentes (G. Lakoff, 1980). Por ejemplo, en el caso que nos ocupa, las metáforas conceptuales RESTRICCIONES=CONTENEDOR, CUERPO HUMANO=CONTENEDOR pueden explicar el significado de salirse de madre, si pensamos que la madre puede asimismo ser la representación metafórica de la educación. Considérese asimismo la expresión desmadrarse. Es preciso aclarar, no obstante, que en este trabajo me he desentendido del significado etimológico de estas expresiones.
} 
Asimismo, conviene destacar aquí que el hecho de que en estas expresiones el verbo salir aparezca en su forma pronominal no es fortuito sino que tiene su explicación en términos de la Estructura Eventiva del evento denominado ${ }^{12}$. El subevento enfocado por el pronombre es la fase de estado que sigue al subevento culminante L: T2 (L3 (L, P), L2 $(\mathrm{L}, E))$.

Así pues, las expresiones salir(se) por [peteneras/la tangente] juntan las dos posibilidades: en la forma sin se, las palabras que denotan manera de desplazarse enfocan la fase de proceso $\mathrm{P}$, dando al evento la configuración aspectual T2 (L3 (L, P), L2 (L, E)); en la variante con se, el clítico aspectual fuerza el enfoque de la fase de estado que sigue al punto culminante, equivalente a «estar ya apartado» del asunto y el sintagma por enfoca la fase de proceso. La configuración es, pues, T2 (L3 (L, P), L2 (L, E)) $)^{13}$.

\subsection{Salir como «aparecer»}

El significado de las expresiones del Grupo C se podría parafrasear grosso modo como «aparecer», «hacerse público» o incluso «surgir», significado que, así como es característico de la mayoría de las expresiones idiomáticas, no se puede deducir sumando los significados de las partes constituyentes de la expresión.

No obstante, si aceptamos que el significado básico de salir es «pasar de un lugar delimitado a otro no delimitado» y que ese lugar delimitado es percibido a menudo como escondido o invisible, la interpretación metafórica de salir como «dar el paso de no estar a la vista a estar a la vista», es decir, «aparecer», «surgir» o «dar a conocer», se vuelve más directa.

Desde esta perspectiva, sintagmas como al aire, a la luz, a la arena, en la tele completan la información aportada por salir; se debe tener en cuenta que no solamente el verbo salir tiene una definición infraespecificada y por tanto potencial significativo sino que todos los componentes de una combinación tienen sus propios aspectos esenciales -qualia-que les hacen susceptibles de interpretarse metafóricamente de formas muy diversas.

La transición tiene, pues, como subevento prominente la fase de logro, el punto culminante: T2 (L3 (L, P), L2 $(L, \mathrm{E}))$.

\subsection{Salir como «resultar»}

Las expresiones recopiladas en el Grupo D representan quizá el mejor argumento a favor de la hipótesis de que el verbo salir tiene en estas combinaciones de palabras muy poco significado: puede ser perfectamente sustituido por otros verbos como quedar, acabar/terminar sin alterar el significado global de las mismas. Su interpretación es muy próxima a «resultar» - en algunas ocasiones puede incluso ser sustituido por resultar en el sentido de que expresa el resultado de un proceso, su finalidad, su término o su conclusión.

\footnotetext{
${ }^{12}$ De acuerdo con De Miguel y Fernández Lagunilla (2000a), quienes proponen que el pronombre clítico es un operador aspectual que señala que el evento culmina en un punto que da lugar a un cambio de estado y lo denominan «se aspectual» o «clítico culminativo».

${ }^{13}$ Un análisis sobre adverbios que enfocan una fase del evento, mientras que otros operadores enfocan otra fase del mismo evento, se encuentra en Fernández Lagunilla y De Miguel (2000).
} 
Las palabras que lo acompañan suelen expresar, literal o metafóricamente, esta valoración del resultado: bien/mal, ganando/perdiendo, rana, escaldado, tarifando. En otras expresiones el verbo salir es seguido por un SP o un SN que no son más que la representación metafórica de una situación prototípica negativa o positiva: con la cabeza caliente y los pies fríos, la criada respondona, el tiro por la culata, a pedir de boca etc.

En este caso, se podría argumentar además, en términos de Pustejovsky, que es la información aportada por los complementos de salir - de cómo o cuál es el final del proceso - la que le da a salir el significado de «resultar», seleccionando el quale TÉLICO del verbo - aquello que codifica el propósito específico de la actividad, como se recogía en el apartado 1.2. No obstante, queda todavía por investigar la relación entre los demás significados de salir y sus qualia, lo que constituye el objeto de otra investigación (Radulescu, en preparación). La configuración aspectual del verbo puede ser, pues, T2 (L3 $(\mathrm{L}, P), \mathrm{L} 2(\mathrm{~L}, \mathrm{E}))$.

\subsection{Salir en expresiones idiomáticas en inglés y rumano}

Las expresiones idiomáticas o metafóricas que, en inglés o en rumano, tienen la misma interpretación o transmiten la misma información que las expresiones en español tienden a usar mucho menos el verbo equivalente, a saber, come out -para el inglés- y a iesi -para el rumano. En (15) y (16) he recopilado algunas de las expresiones formadas con estos dos verbos y también otras expresiones que hacen uso de verbos y procedimientos distintos (pero aspectualmente relacionados) para expresar lo mismo que el verbo salir en español.

turn out [well/all right]
go off at atangent
turn out perfectly
work out expensive
come out of the abyss
come out of a jam
come out ahead
lose out
come out well
come what may

(16)

a iesi la liman
a o scoate la capat
a iesi din incurcatura
a iesi pe dos
a iesi la fix
a se scoatela scapa de saracie
a iesi in castig
a se scoate
a iesi in pierdere
a iesi la iveala

salir a pedir de boca

salir(se) por la tangente

salir redondo

salir caro

salir del agujero

salir del apuro

salir ganando

salir perdiendo

salir bien

a lo que salga

salir a flote

salir adelante

salir del paso

salirle el tiro por la culata

salir redondo

salir del agujero

salir ganando

salir ganando

salir perdiendo

salir a la luz. 
Aunque por razones de espacio el conjunto de expresiones en inglés y rumano es muy limitado, las que he recopilado nos permiten una aproximación al fenómeno que estoy analizando: tanto en inglés como en rumano, el verbo salir, es decir, come out y a iesi, mantiene los mismos significados que en español y entra como constituyente en construcciones muy similares desde el punto de vista de su interpretación idiomática.

En inglés, se observa no ya la preferencia por el verbo come out, de por sí un verbo complejo que equivale a salir pero que está formado por un verbo que significa «venir» y una partícula adverbial que significa «fuera», sino la preferencia precisamente por esa partícula adverbial en combinación con otros verbos, work, lose, turn, get. Asimismo, no es infrecuente, junto con estos y otros verbos, el uso de otras partículas adverbiales o preposicionales que indican un movimiento hacia fuera o hacia arriba, up, off, away.

Además, es posible incluso encontrar en inglés construcciones que prescinden de cualquier verbo para expresar el mismo contenido idiomático solamente con la partícula adverbial out. Por ejemplo, out of the frying pan into the fire, equivalente a la expresión española no salir de una para entrar en otra. Conviene resaltar aquí que tanto en español como en inglés y en rumano, existen numerosas construcciones idiomáticas formadas con el verbo entrar (y sus equivalentes) que tienen un significado global simétrico al de las expresiones formadas con salir. En otras palabras, el verbo entrar tiene un comportamiento semántico muy próximo al de salir con el que comparte el alto grado de infraespecificación y la profusión combinatoria ${ }^{14}$.

En rumano, las expresiones metafóricas o idiomáticas con interpretación similar a las expresiones españolas suelen ser formadas con el verbo salir ( $a$ iesi), al igual que en español. No obstante, hay algunos verbos más que se pueden usar como, por ejemplo, librarse (a scapa) o rebosar (a da pe afara $)^{15}$.

Asimismo, se observa en las construcciones en rumano un frecuente uso del verbo sacar (a scoate) relacionado aspectualmente con verbo salir y que también se usa en español en contrapartida: sacar los pies del [tiesto/plato\} y salirse del tiesto -que equivale a sacar los pies de las alforjas.

Por consiguiente, se puede concluir que los tres idiomas considerados, el español, el inglés y el rumano, se valen de procedimientos muy similares para expresar el mismo contenido idiomático y que el verbo salir demuestra cierta simetría desde el punto de vista de su comportamiento semántico.

\section{Conclusiones}

Sin duda, existen muchas expresiones con el verbo salir que no han tenido cabida en este trabajo y queda por investigar la recuperación de todos los significados del verbo, literales o idiomáticos, y también la relación entre salir y entrar, verbo con el cual presenta mucha simetría, como constituyente de muy diversas expresiones idiomáticas.

No obstante, en este trabajo se ha intentado estudiar el significado del verbo salir en expresiones idiomáticas, partiendo de la hipótesis de que necesariamente ha de tener un significado muy débil. He propuesto una definición básica y comprobado cómo cada una

\footnotetext{
${ }^{14}$ Véase Radulescu (en preparación).

${ }^{15}$ Conviene resaltar que estos verbos no son más que equivalentes a los significados de salir en muchas expresiones españolas. El verbo salir tiene, por ejemplo, el significado de «rebosar» en las expresiones está que se sale o salir(se)le por las orejas.
} 
de las palabras componentes, con su potencialidad significativa, aporta la información necesaria para descodificar el significado global de las expresiones, por muy opaco que éste parezca en ocasiones. Esto se debe a las valencias sin rellenar que cada una de las partes constituyentes de la expresión tiene y a los adecuados mecanismos que permiten completarlas cuando se combinan los constituyentes. Información y mecanismos que, en la hipótesis que sustenta este trabajo, todos los hablantes poseen y no son, pues, privativos de una lengua.

\section{BIBLIOGRAFÍA}

Bosque, I. (2001): «On the weight of light predicates». En: Features and Interfaces in Romance. Herschenson, J.; Zagona, K. y Mallén, E. (eds.). Amsterdam: John Benjamins Publications, 23-38.

Cifuentes Honrubia, J.-L. (1999): Sintaxis y semántica del movimiento. Aspectos de gramática cognitiva. Alicante: Instituto de Cultura "Juan Gil-Albert".

Climent Roca, S. (2000): «Individuación e información Parte-Todo. Representación para el procesamiento computacional del lenguaje». En: Estudios de lingüística española, 8.

Dobrovol'skij, D. (2000): «Idioms in Contrast: A Functional View». En: Las lenguas de Europa: estudios de fraseología, fraseografía y traducción. Corpas, G. (ed.). Granada: Comares 2000, 367-388.

Fernández Lagunilla, M. y De Miguel, E. (2000): «Adverbios de manera e información aspectual». En: Actas del IV Congreso de Lingüística General. Cádiz: Universidad de Cádiz, 1009-1019.

Glucksberg, S. (2001): Understanding Figurative Language- From Metaphor to Idioms. Oxford: Oxford University Press.

Koike, K. (2001): Colocaciones léxicas en el español actual: estudio formal y léxico-semántico. Madrid: Takushoku University, Alcalá de Henares Universidad.

Lakoff, G. y Johnson, M. (1980): Metaphors We Live By. Chicago: The University of Chicago Press.

Miguel, E. de (1999): «El aspecto léxico». En: Gramática descriptiva de la lengua española. Bosque, I. y Demonte, V. (ed.). Madrid: Espasa-Calpe, 2977-3060.

Miguel, E. de (2004a): «La formación de pasivas en español: análisis en términos de la Estructura de Qualia y la Estructura Eventiva». En: Verba Hispánica, XII, 107-129.

Miguel, E. de (2004b): «Qué significan aspectualmente algunos verbos y qué pueden llegar a significar». En: Estudios de Lingüística: el verbo, número monográfico de ELUA. Cifuentes, J.-L. y Marimón, C. (coord.). Universidad de Alicante, 167-206.

Miguel, E. de (2005): «El peso relativo de los nombres y de los verbos: cambios, ampliaciones, reducciones y pérdidas de significado verbal«. En: Homenaje a Ramón Santiago (en prensa). Puigvert, A. et al. (eds.).

Miguel, E. de y Fernández Lagunilla, M. (2000a): «El operador aspectual se». En: Revista Española de Lingüística, 31, 1, 13-43. 
Miguel, E. de y Fernández Lagunilla, M. (2000b): «Predicación secundaria y modificación adverbial». En: Actas del II Congreso de la Sociedad Española de Lingüística: Madrid, Gredos, 226-233.

Miguel, E. de y Fernández Lagunilla, M. (en prensa): «Sobre la naturaleza léxica del aspecto composicional». En: Actas del VI Congreso de Lingüística General. Santiago de Compostela: Universidad de Santiago de Compostela.

Pustejovsky, J. (1993): «Type Coercion and Lexical Selection». En: Semantics and the Lexicon. Pustejovsky, J. (ed.). Dordrecht: Kluwer Academic Publishers, 73-94.

Pustejovsky, J. (1995 [1992]): «The Syntax of Event Structure». En: Lexical and Conceptual Semantics. Levin, B., y Pinker, S. (eds.). Cambridge: Blackwell, 47-81.

Pustejovsky, J. (1998 [1995]): The Generative Lexicon. Cambridge: MIT Press.

Pustejovsky, J. (2000): «Events and the Semantics of Opposition». En: Events as Grammatical Objects. Tenny, C. y Pustejovsky, J. (eds.). Stanford: CSLI Publications, 445-482.

Radulescu, R. (2004): Estudio contrastivo de expresiones idiomáticas del campo de la Conversación en español, inglés y rumano. Trabajo de Investigación Tutelado inédito. Universidad Autónoma de Madrid.

Radulescu, R. (en prensa): Estudio contrastivo de expresiones idiomáticas en español, inglés y rumano. Peter Lang Publishers.

Radulescu, R. (en preparación): Los verbos salir y entrar en expresiones metafóricas e idiomáticas.

Radulescu, R. (en preparación): ¿Qué significa el verbo salir?.

Ruíz Gurillo, L. (2001): «La fraseología como cognición: vías de análisis». En: Lingüística Española Actual. XXIII (1), 107-132.

Serradilla Castaño, A. (2004): «Ir y caer como constituyentes de locuciones fraseológicas que no implican movimiento». Verba Hispánica. XII, 131-141.

\section{IDIOMATIČNE ZVEZE Z GLAGOLOM SALIR V ŠPANŠČINI, ANGLEŠČINI IN ROMUNŠČINI}

Namen pričujoče študije je analiza semantičnega obnašanja glagola salir $\mathrm{v}$ različnih besednih kombinacijah (npr. salir a flote, salir adelante ali salir a pedir de boca) in prikaz podobnosti in razlik pri ustreznih metaforičnih izrazih $\mathrm{v}$ angleščini in romunščini (come out of a jam, come out ahead, turn out, v angleščini; a ie $i$ la liman, a ie i din impas, $\mathrm{v}$ romunščini).

Raziskava se loteva metaforičnih ali idiomatičnih izrazov z vidika Generativnega leksikona Jamesa Pustejovskyja, ki meni, da imajo besede infradoločeno definicijo, opredeljeno s štirimi bistvenimi vidiki, ki s pomočjo generativnih mehanizmov, ki delujejo nanje, omogoča priklic različnih pomenov. Članek poglablja to védenje in želi dokazati, da metaforični pomeni glagola salir v idiomatičnih zvezah dejansko nastajajo prek enakih mehanizmov, kot se to dogaja pri prostih kombinacijah besed, in napoveduje, da so ti mehanizmi splošno veljavni za več jezikov. 